\title{
The Evolution of the Use of Serum Alpha-fetoprotein in Clinical Liver Cancer Surveillance
}

\author{
Sarah-Louise Kelly ${ }^{1}$ and Thomas G Bird ${ }^{1,2^{*}}$ \\ ${ }^{1}$ Centre for Liver and Digestive Disorders, Royal Infirmary of Edinburgh, UK \\ ${ }^{2}$ Cancer Research UK, Beatson Institute, Glasgow, UK
}

"Corresponding author: Bird TG, Centre for Liver and Digestive Disorders, Royal Infirmary of Edinburgh, Edinburgh, EH16 4SA, United Kingdom, Tel: 07890158877; Email: t.bird@beatson.gla.ac.uk

Received date: December 02, 2016; Accepted date: December 31, 2016; Published date: December 31, 2016

Copyright: @ 2016 Kelly SL, et al. This is an open-access article distributed under the terms of the Creative Commons Attribution License, which permits unrestricted use, distribution, and reproduction in any medium, provided the original author and source are credited.

\begin{abstract}
Liver cancer is the $6^{\text {th }}$ most common cancer and $2^{\text {nd }}$ leading cause of cancer-related mortality. In order to improve patient survival early tumor detection is required and this necessitates accurate screening of at risk individuals. In this article we concisely review the methodologies employed for Hepatocellular Carcinoma (HCC) surveillance and how their use has evolved over the last three decades. We focus attention to serum biomarkers, particularly alphafetoprotein. We propose that by using an increasingly sophisticated approach to assess dynamic rates of change in biomarkers tailored to individual patients that screening accuracy may be improved. Additional improvements may also be possible by the incorporation of patient clinical data into such personalised screening assessments. These possibilities may hold the promise of improving cancer detection and early curative therapy for the increasing worldwide population at risk of HCC development.
\end{abstract}

Keywords: Hepatocellular carcinoma; Cancer screening; Alphafetoprotein

\section{Short Communication}

Liver cancer poses an enormous and increasing burden upon human health worldwide. Hepatocellular carcinoma (HCC) is the $6^{\text {th }}$ most common cancer and $2^{\text {nd }}$ leading cause of cancer-related mortality [1]. Crucially, cancer incidence and mortality continue to rise and increasingly affect a younger population [2].

Primary liver cancer occurs in two main forms. The first, Hepatocellular Carcinoma (HCC), accounts for $70-90 \%$ of all primary liver cancer worldwide and represents a tumour formed from hepatocytes within the liver. The other form is Intra-hepatic Cholangiocarcinoma (ICC); a cancer of the bile ducts, which represents $10-25 \%$ of primary liver cancers in most countries worldwide $[3,4]$. The exception to this however is Thailand, where cholangiocarcinoma is the predominant type due to the high incidence of liver fluke infection, which is a major risk factor for ICC development. This article will focus upon HCC, since it is the more treatable form, and has a strong association with an identifiable population at high risk. HCC therefore represents an excellent target for cancer screening.

The strongest risk factor for HCC development is cirrhosis of any cause and the majority of HCCs develop in patients with cirrhosis [5]. As symptoms from HCC develop typically once the disease has advanced beyond the stage of effective or curative therapy, screening of asymptomatic at risk patients has the possibility of detecting treatable tumours and improving patient outcomes. This was demonstrated in the only randomised controlled trial of screening for HCC, involving over 18,000 Chinese patients with hepatitis B. Using six-monthly bimodal screening comprising liver ultrasound imaging and the serum biomarker AFP (alpha-fetoprotein), Zhang et al. demonstrated a reduction in HCC mortality of $37 \%$ [6].

Since this study, and despite intensive research and debate, little consistency in screening practices has been achieved [7]. The cost effectiveness of HCC screening is disputed, and the efficacy of screening tests remains poor. Recent studies have supported the role for HCC surveillance and support its cost effectiveness $[8,9]$. Nonetheless the imperfections of HCC screening, combined with low rates of enrolment of at risk patients into HCC screening, mean that many patients develop HCC having missed the window in which their tumour may have been effectively treated. Treatment options may be curative either through surgical removal of the tumour (by resection or orthotopic transplantation) or loco-regional therapy, however many loco-regional options or pharmacological therapies are aimed at delaying rather than curing the disease [10].

For the purpose of this discussion we will focus on how HCC surveillance should be performed rather than in which target population: principally patients with cirrhosis, or at high risk due to family history, significant fibrosis associated Hepatitis $C$ virus infection or selected patients with Hepatitis B virus - for detailed guidance in this we refer the reader to guidelines [11,12]. Diagnosis of HCC may be made without tissue biopsy as cross-sectional imaging using contrast enhanced CT or MRI has been shown to be accurate in HCC discrimination. Nonetheless, neither imaging modality lends itself as a screening test due to a combination of factors including cost, radiation exposure, claustrophobia and the detection of indeterminate lesions. Hence, other tests are typically used for initial screening to identify patients with probable HCC. Cross sectional imaging is then used to confirm or refute the diagnosis. Liver ultrasound is the imaging modality of choice in screening but is often supplemented by the use of serum biomarkers with the aim of improving early HCC detection. Initial population screening for HCC was implemented in Japan in 1980s [13], and it is informative to track the consensus opinion offered 
by a variety of guidelines on the recommendations for screening tests for HCC (Figure 1). In the context of such seemingly minor adjustments to screening policies over recent decades, liver cancer incidence has risen dramatically whilst HCC survival has remained dismal. Current international guidelines differ in their recommendations. The major European and US bodies recommend that liver ultrasound alone be used for HCC surveillance $[11,12]$. Other international guidelines recommend the addition of at least one serum biomarker, always including AFP (Figure 1).

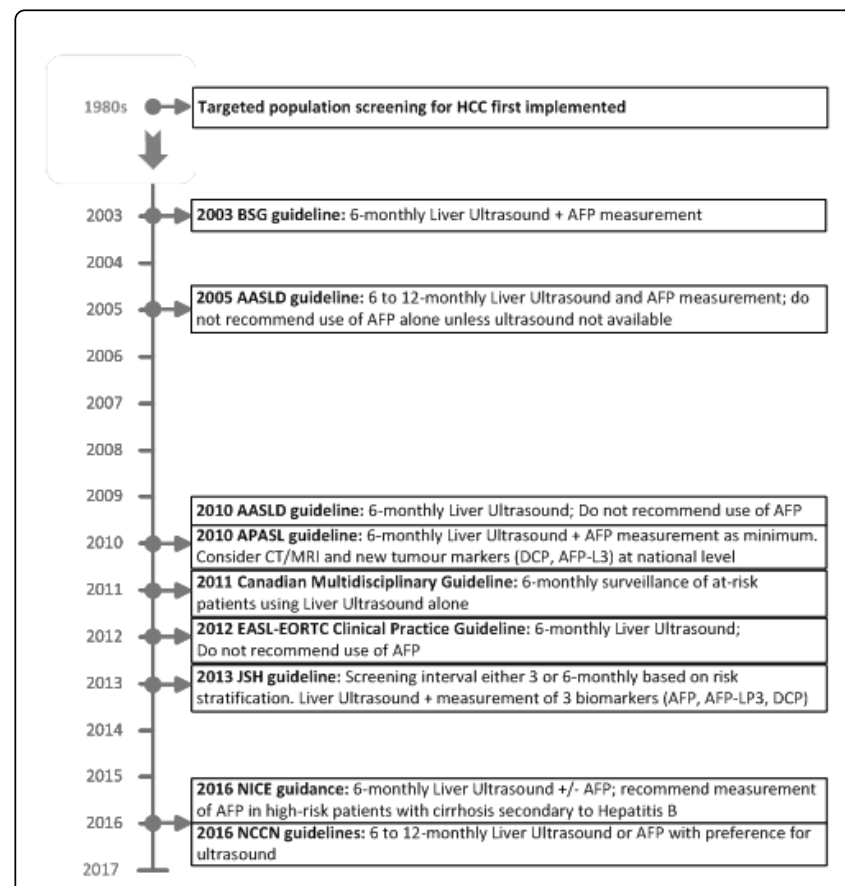

Figure 1: Timeline of HCC surveillance guidelines. Guidance from: BSG: British Society of Gastroenterology [7], AASLD: American Association for the Study of Liver Disease [14], APASL: Asian Pacific Association for the Study of the Liver [15], Canadian Multidisciplinary Guideline [16], EASL: European Association for the Study of the Liver, EORTC: European Organisation for Research and Treatment of Cancer [12], JSH: Japanese Society of Hepatology [17], NICE: National Institute for Health and Care Excellence, UK [18], NCCN: National Comprehensive Cancer Network, Guidelines Hepatobiliary Cancers [19].

Due to the relative conservation of the molecular phenotype of HCC, it is plausible that a single or small panel of serum biomarkers may detect the overwhelming majority of cancers [20]. Serum biomarkers have a number of clear advantages as a screening tool; they are minimally invasive, can be standardised and are relatively inexpensive. A major ongoing challenge in the field of HCC surveillance is to identify suitable serum markers and validate their appropriate use.

Undoubtedly the best known and most widely used serum biomarker for HCC is Alpha-fetoprotein (AFP). AFP is produced by the embryonic yolk sac and subsequently the foetal liver, with maternal serum levels falling rapidly after birth. Elevated maternal serum AFP levels are used as part of screening for foetal abnormalities including neural tube defects. AFP may be pathologically elevated by the presence of germ cell tumours or HCC [21]. Serum AFP levels may also be raised in chronic liver disease with high levels of hepatocyte regeneration but remain low in the majority of patients with cirrhosis in the absence of HCC. The use of AFP as a tumour marker for HCC was first described in the 1960s [22]. Limitations to efficacy of AFP, particularly with a static cut-off are numerous (Table 1) and summate to a poor sensitivity and specificity when assessed in many clinical trials. For this reason AFP has fallen out of favour as a screening test (Figure 1). Nonetheless, some studies have suggested that AFP may inform clinical decision making and lead to curative therapy of HCC $[23,24]$.

- Substantial proportion of HCCs do not secrete AFP, irrespective of size [25]
- AFP frequently within normal range in small $(<3 \mathrm{~cm})$ HCCs [26]
- Elevated AFP can occur with chronic liver disease in absence of HCC,
particularly Hepatitis C infection $[27,28]$
- Variable sensitivity and specificity depending on the cut-off value used [29]
- Very high AFP is a marker of poor prognosis; identifying patients with
advanced HCC [30]
End result: Poor sensitivity and specificity when tested clinically

Table 1: Limitations to efficacy of AFP

With the movement away from the use of AFP earlier this decade and the absence of alternative serum biomarkers, the frailty of using liver ultrasound alone as a screening test has become an increasing concern. In many studies, the sensitivity and specificity of liver ultrasound have been relatively poor, ranging from $60-90 \%$ and $90-95 \%$ respectively [31-33]. When used in a real world setting, the accuracy has been even worse [34]. Therefore, with this unmet need, there is an increasing drive to develop improved serum biomarkers for use in the growing worldwide population of patients at high risk of HCC development.

There is a possibility, however, that a more sophisticated interpretation of existing biomarkers and the combination of single or multiple biomarker levels with demographic information may significantly improve their accuracy. This is now practically feasible as large datasets exist which can be mined for patterns identifying underlying HCC. For example, numerous studies have now shown that absolute changes in AFP values from a nadir can improve accuracy [35]. Others, including ourselves, have now shown that measuring dynamic rates of change over time for individuals can also be used effectively $[23,36]$. A further strategy has been to combine multiple serum markers. Additional serum markers studied in this way include the AFP-LP3 isoform, Des-gamma-carboxyprothrombin (DCP) and Golgi protein 73 (GP73) amongst others [37]. Meta-analysis of studies in 2013 showed that certain combinations of markers provided greater diagnostic accuracy than each alone [38]. Never the less, variations in biochemical assays and differing cut-offs values applied in different retrospective studies have limited the clinical applicability of using multiple biomarkers in most cases. However one example of a combination of serum biomarkers used synchronously which has moved towards validated clinical practice is by the group of Philip Johnson [39]. They have utilised a panel of three biomarkers (AFP, its AFP-LP3 isoform, and DCP). Importantly Johnson et al. used this marker panel and combined it with other variables (patient age and gender) to create the GALAD score and have now validated this in patient cohorts from Germany, Japan and Hong Kong [40]. The GALAD score has consistently discriminated patients with HCC from 
HCC-free patients in real world screening cohorts, with Area under Receiver Operator Curve values of $>0.90$, and it is now freely available to use via the Mayo Clinic portal. Additional optimisation is still required, particularly for patients with small uni-focal HCCs for whom medical therapy is most likely to be successful. It is the identification of this population which is crucial as recent studies have clearly shown that early detection is associated with broader treatment options and improved survival [8]. It is possible that the further combination of monitoring dynamic changes in these three serum biomarkers and additional clinical information, including disease aetiology, may allow further improvement in the accuracy of serum biomarker detection of HCC.

An ongoing requirement is large well characterised prospectively followed cohorts. Such data are vital to explore and validate ongoing iterative improvements in HCC surveillance programmes. We predict that this will lead to the eventual inclusion of multiple variables, analysed in a dynamic fashion [23], to improve the detection of presymptomatic HCC. It is likely in the short term that serum biomarkers will continue to be used as an addition to ultrasound based screening to identify very high risk patients in whom ultrasound may be unable to detect an early HCC. However in the future serum biomarkers may be used to stratify risk and possibly even negate the need for imaging based surveillance, at least transiently, in highly selected patients. If such strategies prove to be successful then we might see the broad reintroduction of serum biomarkers into increasingly complex clinical guidelines for HCC surveillance.

In conclusion, HCC presents an unmet need in worldwide disease. In order to meet this, screening of asymptomatic patients is required. However, this screening needs to be accurate and cost effective. Despite having fallen out of favour, serum biomarkers may still hold the key to improving HCC screening. Using these simple tests and basic clinical information in an analytically more sophisticated manner, we may make a genuine impact on the increasing mortality rates of HCC worldwide.

\section{Acknowledgement}

TGB is funded by a Wellcome Trust Intermediate Clinical Fellowship (107492/Z/15/Z).

\section{References}

1. International Agency for Research on Cancer (2012) GLOBOCAN 2012: Estimated Cancer Incidence Mortality and Prevalence Worldwide in 2012. World Health Organisation.

2. Ryerson AB, Eheman CR, Altekruse SF, Ward JW, Jemal A, et al. (2016) Annual Report to the Nation on the Status of Cancer, 1975-2012, featuring the increasing incidence of liver cancer. Cancer 122: 1312-1337.

3. Center MM, Jemal A (2011) International Trends in Liver Cancer Incidence Rates. Cancer Epidemiol Biomarkers Prev 20: 2362-2368.

4. Ahmed F, Perz JF, Kwong S, Jamison PM, Friedman C, et al. (2008) National trends and disparities in the incidence of hepatocellular carcinoma, 1998-2003. Prev Chronic Dis 5: A74.

5. Fattovich G, Stroffolini T, Zagni I, Donato F (2004) Hepatocellular carcinoma in cirrhosis: incidence and risk factors. Gastroenterology 127: S35-S50.

6. Zhang BH, Yang BH, Tang ZY (2004) Randomized controlled trial of screening for hepatocellular carcinoma. J Cancer Res Clin Oncol 130: 417-422.

7. Joshi K, Mendler M, Gish R, Loomba R, Kuo A, et al. (2014) Hepatocellular Carcinoma Surveillance: A National Survey of Current Practices in the USA. Dig Dis Sci 59: 3073-3077.
8. Mittal S, Kanwal F, Ying J, Chung R, Sada YH, et al. (2016) Effectiveness of surveillance for hepatocellular carcinoma in clinical practice: A United States cohort. J Hepatol 65: 1148-1154.

9. van Meer S, de Man RA, Coenraad MJ, Sprengers D, van Nieuwkerk KMJ, et al. (2015) Surveillance for hepatocellular carcinoma is associated with increased survival: Results from a large cohort in the Netherlands. J Hepatol 63: 1156-1163.

10. S Au J, T Frenette C (2015) Management of Hepatocellular Carcinoma: Current Status and Future Directions. Gut Liver 9: 437-448.

11. Bruix J, Sherman M (2011) Management of hepatocellular carcinoma: An update. Hepatology 53: 1020-1022.

12. European Association for the Study of the Liver, European Organisation for Research and Treatment of Cancer (2012) EASL-EORTC Clinical Practice Guidelines: Management of hepatocellular carcinoma. J Hepatol 56: 908-943.

13. Mima S, Sekiya C, Kanagawa H, Kohyama H, Gotoh K, et al. (1994) Mass screening for hepatocellular carcinoma: experience in Hokkaido, Japan. J Gastroenterol Hepatol. 9: 361-365.

14. Bruix J, Sherman M (2005) Management of hepatocellular carcinoma. Hepatology 42: 1208-1236.

15. Omata M, Lesmana LA, Tateishi R, Chen P-J, Lin S-M, et al. (2010) Asian Pacific Association for the Study of the Liver consensus recommendations on hepatocellular carcinoma. Hepatol Int 4: 439-474.

16. Sherman M, Burak K, Maroun J, Metrakos P, Knox JJ, et al. (2011) Multidisciplinary Canadian Consensus Recommendations for the Management and Treatment of Hepatocellular Carcinoma. J Curr Oncol 18: 228-240.

17. The Japan Society of Hepatology (2013) Clinical Practice Guidelines for Hepatocellular Carcinoma 2013. Chapter 2: Diagnosis and Surveillance. Section 2: Tumour markers. The Japan Society of Hepatology.

18. National Institute for Health and Care Excellence (2016) NICE Clinical Guideline (NG50) Cirrhosis in over 16s: assessment and management. National Institute for Health and Care Excellence.

19. National Comprehensive Cancer Network (2016) NCCN Clinical Practice Guidelines in Oncology. Hepatobiliary Cancers. Version 2.2016. NCCN.

20. Zucman-Rossi J, Villanueva A, Nault J-C, Llovet JM (2015) Genetic Landscape and Biomarkers of Hepatocellular Carcinoma. Gastroenterology 149: 1226-1239.

21. Ball D, Rose E, Alpert E (1992) Alpha-fetoprotein levels in normal adults. Am J Med Sci 303: 157-159.

22. Abelev GI (1968) Production of embryonal serum alpha-globulin by hepatomas: review of experimental and clinical data. Cancer Res 28 : 1344-1350.

23. Bird TG, Dimitropoulou P, Turner RM, Jenks SJ, Cusack P, et al. (2016) Alpha-Fetoprotein Detection of Hepatocellular Carcinoma Leads to a Standardized Analysis of Dynamic AFP to Improve Screening Based Detection. PLoS One. 11:e0156801.

24. El-Serag HB, Kanwal F (2013) a-Fetoprotein in Hepatocellular Carcinoma Surveillance: Mend It but Do Not End It. Clin Gastroenterol Hepatol 11: 441-443.

25. Ryder SD (2003) Guidelines for the diagnosis and treatment of hepatocellular carcinoma (HCC) in adults. Gut 3: 1-8.

26. Chen DS, Sung JL, Sheu JC, Lai MY, How SW, et al. (1984) Serum alphafetoprotein in the early stage of human hepatocellular carcinoma. Gastroenterology 86: 1404-1409.

27. Di Bisceglie AM, Sterling RK, Chung RT, Everhart JE, Dienstag JL, et al. (2005) Serum alpha-fetoprotein levels in patients with advanced hepatitis C: Results from the HALT-C Trial. J Hepatol 43: 434-441.

28. Di Bisceglie AM, Hoofnagle JH (1989) Elevations in serum alphafetoprotein levels in patients with chronic hepatitis B. Cancer. 64: 2117-2120.

29. Gupta S, Bent S, Kohlwes J (2003) Test characteristics of alpha-fetoprotein for detecting hepatocellular carcinoma in patients with hepatitis C. A systematic review and critical analysis. Ann Intern Med 139: 46-50. 
Citation: Kelly SL, Bird TG (2016) The Evolution of the Use of Serum Alpha-fetoprotein in Clinical Liver Cancer Surveillance. J Immuno Biol 1:

Page 4 of 4

30. Gupta S, Kutty G, Jagar P, Jain P, Lad T (2013) P-0112* very high alphafetoprotein (afp): a poor prognostic indicator in hepatocellular carcinoma in the modern era. Ann Oncol 24: iv67-iv67.

31. Daniele B, Bencivenga A, Megna AS, Tinessa V (2004) Alpha-fetoprotein and ultrasonography screening for hepatocellular carcinoma. Gastroenterology 127: S108-S112.

32. Maringhini A, Cottone M, Sciarrino E, Marcenó MP, La Seta F, et al (1988) Ultrasonography and alpha-fetoprotein in diagnosis of hepatocellular carcinoma in cirrhosis. Dig Dis Sci 33: 47-51.

33. Sherman M, Peltekian KM, Lee C (1995) Screening for hepatocellular carcinoma in chronic carriers of hepatitis $B$ virus: Incidence and prevalence of hepatocellular carcinoma in a North American urban population. Hepatology 22: 432-438.

34. Singal AG, Conjeevaram HS, Volk ML, Fu S, Fontana RJ, et al. (2012) Effectiveness of Hepatocellular Carcinoma Surveillance in Patients with Cirrhosis. Cancer Epidemiol Biomarkers Prev 21: 793-799.

35. Chang TS, Wu YC, Tung SY, Wei KL, Hsieh YY, et al. (2015) AlphaFetoprotein Measurement Benefits Hepatocellular Carcinoma Surveillance in Patients with Cirrhosis. Am J Gastroenterol 110: 836-844.
36. Lee E, Edward S, Singal AG, Lavieri MS, Volk M (2013) Improving Screening for Hepatocellular Carcinoma by Incorporating Data on Levels of a-Fetoprotein, Over Time. Clin Gastroenterol Hepatol 11: 437-440.

37. Van Hees S, Michielsen P, Vanwolleghem T (2016) Circulating predictive and diagnostic biomarkers for hepatitis B virus-associated hepatocellular carcinoma. World J Gastroenterol 22: 8271-8282.

38. Hu B, Tian X, Sun J, Meng X (2013) Evaluation of Individual and Combined Applications of Serum Biomarkers for Diagnosis of Hepatocellular Carcinoma: A Meta-Analysis. Int J Mol Sci 14: 23559-23580.

39. Johnson PJ, Pirrie SJ, Cox TF, Berhane S, Teng M, et al. (2014) The Detection of Hepatocellular Carcinoma Using a Prospectively Developed and Validated Model Based on Serological Biomarkers. Cancer Epidemiol Biomarkers Prev 23: 144-153.

40. Berhane S, Toyoda H, Tada T, Kumada T, Kagebayashi C, et al. (2016) Role of the GALAD and BALAD-2 Serologic Models in Diagnosis of Hepatocellular Carcinoma and Prediction of Survival in Patients. Clin Gastroenterol Hepatol 14: 875-886. 